\title{
BASIC PROCESSES IN SOLID AND LIQUID INSULANTS
}

A ONE-DAY conferenco with the above title, arranged by the Sixth Soction of the Société Française des Electricions and the Diolectric Research Department of the Laboratoire Contral dos Industries Électriques was held in Fontonny-Aux-Roses (Seine) on March 19. The aim of this conferonce was to bring out recent advaneos in the study of dielectric phonomena, including work on the various processes of polarization; transiont, steady, transverse and surface conductivities; phenomena localizod at metal-insulation interfaces and 'intrinsie' diolectric breakdown. All the papers presented at the conferonce doalt with phenomena in solid dielectrics, excopt ono which described recent work at one establishment in the United Kingdom on dielcetric phenomena in mineral oil.

In this report wo shall give a brief résumé of the papers. some of which are to bs published in full in $t a$ Revuse Générale de l'Electricité, because the attondance at tho conference was limited and it is folt that the subjects discussed are of great topical interest to the many people at presont investighting dielectric phenomena.

The conferenco opened with a roview paper by Coelho ("Prosent Problems in the Field of Insulators and Dielectrics") on recent investigations with dioloctrics at very low tcrmperatures. This included work carried out at other laboratorics on the dieloctric properties of liquid helium as well as that on the dielectrio strength of vory pure crystals at low temperatures. The discussed the work of Kühn and Luty of Stuttgart on the paraelectric behaviour of (OH)- hydroxyl dipoles in a $\mathrm{KCl}$ erystal, in which they showed that at an applied field of $10^{7} \mathrm{~V} / \mathrm{m}$ and a tomperature of a few degrees Kolvin it was possiblo to orientate almost all the $(\mathrm{OH})^{-}$dipoles in a diroction parallel to the field.

Fallou and Bouvier ("Fxperiments on tho Conductivity of Polymers in Thin Films") reported measurements of the variation in conduction eurront with film thickness and mean applied gradients for two types of polymer: polyamide 6-6 and polycarbonate. In tho case of polyamide 6-6 they found a considerablo increase in the conductivity with decrease in film thickness. The dielectric losses, when msasured at a low frequency, exhibited a similar but less pronounced variation. However, at higher froquencies the losses were independent of the film thickness. This, Fallou and Bouvier attributed to ionic charges associated with the presence of moisture in the films. Moasuremonts mado on polycarbonate, which was woll dried, did not indiente any variation of conductivity with thicknoss, thus confirming the investigator's hypothesis. For both polymers tho conductivity was indopondent of the mean applied gradient up to $5 \times 10^{4} \mathrm{~V} / \mathrm{cm}$. The dynamies of moving ions, accumulated in surface potontial barriers, and of the carriers exchanged betwoon the surface and the volume of tho dielectric material were discussed in a paper by Goffaux ("Mechanism of Conduotion and Elcctrical Properties of Solid Insulating Materials"). He investigated the variation in loss angle of mica and tri-acetate of cellulose with voltage, frequoncy and tomperature. Goffaux also analysed surface conductivity, taking into consideration tho transfer of surface changes from place to place by a procoss of thermal excitation.

Bucci, Fiosuhi und Guidi ("Ionic Conductivity in Dielectrics") extendod their previous investigntions on dielectric polarizntion using an ionic thermocouple. Such a method depends first on polarizing the sample in a static electric fiold for a given time at a given tempornture. The solid is then cooled down to a temperaturo where any ionic motion is stopped and the extornal field taken off. The solid is then warmed up at a constant rate and the discharge current noted as a function of temperature. The processes of relaxation give rise to bands of current, the analysis of which gives information on the charactoristics of relaxation. For example, using this method in the case of dipolar orientation, the number of dipoles, the activation energy and the parameters of the relaxation can be measured independent of the temperature. Bucei et al. reported several measuroments, particularly on alkali halogenated erystals, where they observed: relaxation of dipoles; strong bands of thermal conductivity (probably due to surface effects), relaxation of space churge and the effect of X-ray irradiation. The capacity of a $\mathrm{Si}-\mathrm{SiO}_{2}-\mathrm{Al}$ structure was measured by Forlani and Minnaja ("Now Methods for the Study of the Interface $\mathrm{Si}_{-} \mathrm{SiO}_{2}$ ") in order to obtain information on the distribution of the charged states at the interface of the silicon. By illuminating the structure they obtained the field distribution betwon the somi-conductor and the dielectric, Conduetion phenomena were also studind by applying a direct voltage botween the silicon and the aluminium, and the distribution of the charged states at the $\mathrm{Si}-\mathrm{SiO}_{2}$ interface deduced from an analysis of the conduction characteristics. Additional information was obtained by analysing the conduction measurements under illumination and thus these investigators wero able to prosent a picture of the phenomena present at the interface $\mathrm{Si}-\mathrm{SiO}$ a under the effect of an applied field.

A review of the various ideas and concepts adopted by the different schools of thought on dielectric losses in organic compounds was given in a review papex by Freymann ("Progress Made Through the Idea of System Faults and the Study of Dielectric Proporties"). Ho discussed the effects of lattice faults and chemical impurities and reported on sorne experimonts from the work of Mme Froymann and Mmo Jaffrain which show that it hydrogen connexion is not indispensable for observing dipolar adsorption. Petiau and Curien ("Studies of the Segregation of the Impuritios in Alkaline Halogenides by Dielectric Measurements") reported investigations of the kineties of the spontaneous variation of the number of dipoles, caused by the prosence of divalent impurities at atomic concentrutions of the order of $10^{-4}$, in halogenated alkalis. Those investigations wore made by the measurement of the dielectric losses at various temperatures between $50^{\circ}$ and $200^{\circ} \mathrm{C}$. They found that a reaction of the third order occurred, possibly duo to a regrouping of the dipoles in trimers, and then a second reaction due to moro complex associations. In crystals with a higher impurity content, of the order of $10^{-2}$, the divalent impurities in $\mathrm{LiF}$ precipitated, in the form of $\mathrm{MgF}_{2}$ or $\mathrm{Li}_{6} \mathrm{MgF}_{8}$, both phases being orientated in the LiF matrix. Petian and Curien studied these precipitations by $\mathrm{X}$-ray diffraction methods and mierography.

A theoretical consideration by Desbrandes ("Theoretical Study of the Vibrational Interactions on Motal-dielectric Contact") of thermal vibrations at the boundary of a erystal in equilibrium with the ambient medium led him to conclude that the alterations of the phonon spectrum at the boundary of a solid characterize the vibrational properties of the ambient medium.

Arnoult, Sixous and Dansas "Froquency Absorption Spectra of Layored Hetcrogeneous Dielectrics; Interface Polarization") investigated, over a range of frequenciss (from a fow c/s to the megacycle range), how the complex permittivity of an assembly of two parallel layer thin sheets is modified when the complex permittivity or the conductivity of either sheet is varied or the ratio of the thickness of the sheets modificd. Using arrangements of mica in conjunction with ethrnol +1 per cent water, and 
propylene glycol $+\mathbf{0 . 5}$ per cent or 3 per cent of a standard soda solution, they studied the variation of the conductivity of the latter substances with temperature. Again, using mica in conjunction with different metal oxide powders (alumina, zine oxide, mercury oxide, etc.), they found that the relationship between the permittivity factor $\varepsilon^{\prime}$ and the loss factor $\varepsilon^{\prime \prime}$ in the Cole-Cole diagram was not a semicircle but rather an arc of a circle the centre of which was below the axis of the abscissa. Lacoste, Letureq and Martinot "Methods of Studying Slow Polarization Phenomena") developed a simple method, based on obtaining three values on the dielectric hysteresis cycle of a material, which makes it possible to calculate immediately $\varepsilon^{\prime}$ and $\varepsilon^{\prime \prime}$ in the frequency range $10^{-1}-10^{-4} \mathrm{e} / \mathrm{s}$. They obtained the frequency response for various insulations (mica, muscovite, lac, methyl. phenyl-polysiloxane, etc.) and reported variations of from 1 to 10 of $\varepsilon^{\prime}$ and $\varepsilon^{\prime \prime}$ which appeared between $10^{-2}$ and $10^{-4} \mathrm{c} / \mathrm{s}$. They also found that $\varepsilon^{\prime \prime}$ plotted as a function of $\varepsilon^{\prime}$ produced one or more ares of a circle centred below the axis of the abscissa indicating a spectra of relaxation comprising time constants distributed in accordance with a Cole-Cole distribution.

In the only paper dealing with liquid dielectries, Nosseir, Hawley and Lawrence ("Recent Research on Fundamental Processes in Liquid Dielectrics under the Influence of High Electrical Stresses") reported results of their investigations of the effects of additives on electrical phenomena in mineral oil. They measured the gassing coefficient and the breakdown strength for oils with different aromatic contents. Increasing the aromatic content in the range 0-25 per cent increased the breakdown voltage and gave a corresponding reduction in the gassing coefficient. A linear relationship was found between gassing coefficient and aromatic content. They also studied the effect of adding different amounts of quinone to a given oil and found that there was an optimum concentration which gave maximum electrical strength; however, this concentration was slightly less than that which resulted in the minimum gassing rate. Nosseir et al. also investigated the effect of additions of sulphur hexafluoride $\left(\mathrm{SF}_{6}\right)$ to mineral oil. It was found that stress conditioning an oil sample containing $\mathrm{SF}_{6}$ caused the breakdown strength to fall and the conduction current magnitude to increase. This was explained by the deposition of sulphur on the electrodes. After a period of stress conditioning luminescence occurred near the cathode. The breakdown strength was dependent on the partial pressure of dissolved $\mathrm{SF}_{6}$. Additions of minute quantities of $\mathrm{SF}_{6}$ resulted in an increase in the electrical strength while further additions decreased the strength. Visual observations indicated that complete breakdown of an oil sample containing $\mathrm{SF}_{6}$ was preceded by initiating events at the anode so that breakdown sparks started at the anode and proceeded to the cathode. Visual observations of a gas/oil interface in the vicinity of an oil gap, stressed by a direct voltage, revealed some interesting phenomena. It was found that with voltage increase the free surface was disturbed sufficiently to give rise to vortices in the liquid which were eventually drawn to one of the electrodes. A. Nossem

R. HAWIEY

\section{THE CARNEGIE UNITED KINGDOM TRUST}

$\mathrm{T}$ THE annual report of the Carnegie United Kingdom Trust*, covering the year 1964, includes tributes to the work of two senior trustees who died during the year, Sir George Dyson and Sir Hector Hetherington.

Grants for the arts at $£ 27,568$ remained much the same as for the previous year, but settlement of the grant of $£ 30,000$ towards the cost of Balvicar Street Centre, Glasgow, for handicapped children brought the total for community services to $£ 78,598$, compared with $£ 31,800$ in 1963 . Grants for education, including $£ 38,668$ for youth services in place of $£ 15,393$ in 1963 , increased to $£ 47,695$. Grants to museums continued on the usual lines and fourteen museums took advantage of the provision for obtaining expert reports on their own organization. Measures that would lead to improving their services were started with the help of Trust funds in five museums, and sixteen

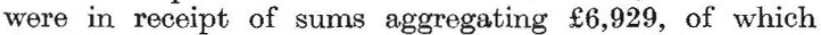
$£ 5,504$ represented final instalments of grants to some twelve museums. A grant of $£ 1,000$ to the Museums Association gave some stability to its financial structure at a time when it was being seriously threatened by rising costs.

Of three grants in 1964 for new communities, one was the provision of a new building with an unusual range of functions in the re-developed area of Scotswood, Nowcastle upon Type; the second was for projects in new communities, for the University Settlement in Barton Hill, Bristol; and the third (and largest) was promised to the Skelmersdale Development Corporation, which will receive $£ 15,000$ towards the capital cost of a social centre at Grimshaw Delf. Two books arising out of the pioneer Bristol Social Project were published. The first, Stress and Release in an Urban Estate, describes work carried out with various groups, including mothers, teachers, social workers, and a mixed gang of adolescents; and a second volume, Life on the Outskirts, a study of neighbourhood

* 'The Carnegie United Kingdom Trust. Fifty-first Annual Report, 1964. Pp. viii $+68+2$ plates. (Dunfermline: The Carnegie United Kingdom Trust, 1965.) and family, was in preparation. During the year, $£ 2,000$ went to the Family Welfare Association towards the initial cost of re-organization, and a report published in June, Handicapped Children and their Families, describes the work of three teams of investigators in Glasgow, Sheffield and Shropshire, who studied the problems of 600 handicapped children and their families.

The Trust again supported the work of the four National Councils of Social Service in England, Scotland, Wales and Northern Ireland, with grants for general purposes, but the only village hall transactions in the past year or two were concerned with halls existing or projected in Eire. The Trust promised a grant of $£ 30,000$ to enable the National Association of Youth Clubs and the Edueation Department of the West Riding of Yorkshire County Council to collaborate in an educational experiment of a pioneer kind in a new secondary school; the grant, which would be a contribution towards the total cost of the school, would be used for building a centre for young people in their last years at school and afterwards. Three other projects for the benefit of difficult young people were the subject of new grants in 1964. The first was concerned with an attempt by the Christian community in the University of Edinburgh to meet the needs of young men who were trying to regain a place in society after a term in borstal or prison. The second went to the Scottish Association of Youth Clubs for a project to attract unattached young people living in a group of housing schemes on the outskirts of Edinburgh. The third was awarded to the National Association of Youth Clubs for its attempt to divert the energies of young people who have been responsible for Sunday riots at numerous seaside resorts.

The Trust was confident that at the end of the year satisfactory arrangements would be made to complete the scheme for the first public nature centre at Brantwood, near Coniston, in 1965. Disappointingly slow progress, however, is also reported with regard to the Brecon 